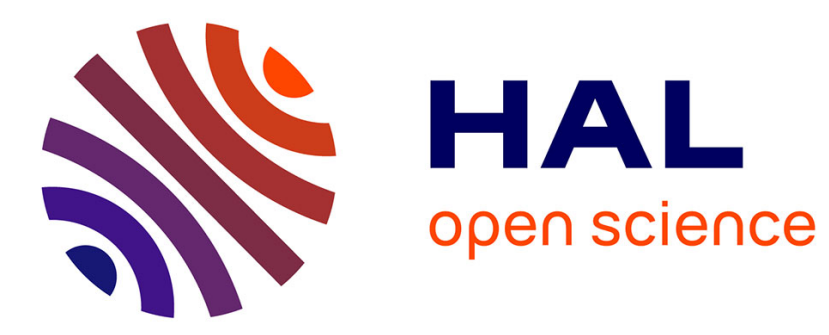

\title{
Etude forestière pédologique et microbiologique d'un bois très dégradé de la Haute-Saône
}

René Schaeffer, R. Moreau

\section{To cite this version:}

René Schaeffer, R. Moreau. Etude forestière pédologique et microbiologique d'un bois très dégradé de la Haute-Saône. Revue forestière française, 1959, 6, pp.417-433. 10.4267/2042/27508 . hal-03382113

\section{HAL Id: hal-03382113 \\ https://hal.science/hal-03382113}

Submitted on 18 Oct 2021

HAL is a multi-disciplinary open access archive for the deposit and dissemination of scientific research documents, whether they are published or not. The documents may come from teaching and research institutions in France or abroad, or from public or private research centers.
L'archive ouverte pluridisciplinaire HAL, est destinée au dépôt et à la diffusion de documents scientifiques de niveau recherche, publiés ou non, émanant des établissements d'enseignement et de recherche français ou étrangers, des laboratoires publics ou privés. 


\section{REVUE FORESTIERE FRANÇAISE}

1959

JUIN

No 6

Dans ce numéro: R. Schaeffer et R. Moreau: Etude forestière pédologique et microbiologique d'un bois très dégradé de la Haute-Saône. - A. Guichon: L'élaboration et la mise en œuvre d'une politique et d'une législation forestières sont aussi des techniques. - Dr. SaAtçioglu et Dr. Pamay : 20 ans de boisement d'eucalyptus à 'Karabucak, près Tarsus. - M. Duval: Aménagement forestier et progrès technique dans $1^{\prime}$ Ouest au XVIII ${ }^{\theta}$ siècle. .. B. Roux : Les « révélateurs 》 du domaine forestier domanial. - P. Groult : Encore le pin Weymouth. - P. BouvareL: Race ou variation clinale. J. BASTIDE: Un exemple d'efficacité des travaux d'entretien du sol dans les plantations.

\section{ETUDE FORESTIEERE, PÉDOLOGIQUE ET MICROBIOLOGIQUE D'UN BOIS TRÉS DÉGRADÉ DE LA HAUTE-SAONE}

Il existe dans la vallée de la Saône à $30 \mathrm{~km}$ en amont de Gray, un village du nom de Fédry, sur le territoire duquel se trouve un bois particulier dit "Bois du Prieur ». Celui-ci est enclavé dans un massif communal et l'ensemble présente des signes non équivoques de dégradation. Le bois particulier se porte de toute évidence plus mal que les autres parce qu'il a subi des épreuves plus dures.

Son propriétaire actuel n'en tire aucune satisfaction: il constate que la composition du peuplement est défectueuse et sa croissance très faible. La seule inspection du domaine, aussi minutieuse qu'elle puisse etre (elle dure depuis r928), n'apportait aucune explication, par conséquent aucun remède à cet état de choses.

Un examen aussi complet que possible s'imposait, qui fut réalisé récemment; ce sont ses résultats qui sont consignés dans la présente note.

\section{Etude forestière classique}

\section{Conditions ÉCOLOGiQUeS}

Sol. - Le terrain est constitué par un plateau ondulé recouvert d'une épaisse couche d'alluvions anciennes quaternaires; le substratum calcaire n'apparait nulle part et semble être en tout point hors de l'atteinte des racines les plus profondes. Le niveau considéré 
est celui dit " des terrasses inférieures », composé de sables fins ou d'argiles grasses rougeâtres. Ces alluvions anciennes donnent généralement des sols profonds, mais pauvres en principes fertilisants (silice pure et argiles (" mortes )).

Le bois occupe les deux versants d'un petit vallon orienté du Nord-Est au Sud-Ouest; le flanc regardant le N.-O. est recouvert par les argiles, le flanc opposé est entièrement sableux, tandis que dans le thalweg les deux sortes de sédiments se mélangent en donnant un sol plutôt compact et assez imperméable.

Les différences de niveau sont faibles, ne dépassant guère 20 à 25 mètres. L'altitude maximum est d'environ 250 mètres.

Climat. - La vallée de la Saône est très ouverte aux influences rhodaniennes: la température moyenne annuelle est assez élevée $\left(9^{\circ} 7\right)$, le vignoble a été largement développé dans toute la région et il en subsiste d'importants vestiges à Fédry même.

La pluviosité est inférieure à la moyenne de la Haute-Saône: elle était en 1955, année moyenne, de $738 \mathrm{~mm}$ à Ray/Saône. distant (le $5 \mathrm{~km}$.

Quant au microclimat, il présente une insolation forte sur le versant S.F., des gelées printanières dans le vallon, tandis que le versant N.O. est frais.

\section{ACTiOn DE L'IIOMMe}

La vallée de la Saône a toujours constitué un habitat de choix pour l'homme et les vestiges de l'occupation romaine sont nombreux. Le parcours des animaux domestiques (porcs, bêtes à cornes) s'est exercé là comme ailleurs pendant tout le Moyen Age et sans doute jusqu'au xviri ${ }^{e}$ siècle.

L'industrie sidérurgique a pris naissance de bonne heure dans cette région: on relève dans un rayon de $10 \mathrm{~km}$ autour de Fédry les noms de Ncidans-le-Ferroux, Vyt-le-Ferroux, Ferrière-les-Ray, bois des Minières, qui ne laissent aucun doute sur l'ancienneté de cette activité grosse consommatrice de bois de feu. On peut ajouter à ce débouché les bourgs de Ray et de Gray qui recevaient leur affouage par bateau en provenance des forêts situées en amont de ces agglomérations.

Au XvirI $^{\mathrm{e}}$ siècle, la pénurie était devenue tragique et la rivalité entre maîtres de forge, qui s'étaient multipliés, dura tant que le charbon de terre ne fut pas d'un usage courant.

Le bois du Prieur, propriété ecclésiastique jusqu'à la Révolution. bénéficia sans cloute de la tutelle de la Maitrise des Eaux et Forêts qui fonctionna à peu près un siècle (I699-I790).

Soumis pendant ce temps au régime commun imposé aux collectivités, c'est-à-dire le taillis-sous-futaie à la révolution de 25 ans, le bois en question se trouva ensuite exposé à la cupidité conjuguée 
des propriétaires privés et des maîtres de forge: les révolutions de $I_{5} 5$, et même $I_{2}$ ans, n'étaient pas rares au début du XIX ${ }^{e}$ siècle, et les réserves se voyaient bien réduites alors en nombre et en taille. Le déclin de la sidérurgie haut-saônaise força les propriétaires à pratiquer une sylviculture plus conservatrice et vers I925 un matériel assez important existait dans ce petit massif. Une coupe brutale effectuée en I928 le ruina à nouveau; depuis il se repose.

\section{DESCRIPTION DU PEUPLEMENT}

Les Arbres. - L'essence la mieux représentée est le Chêne rouvre (Quercus robur), avec quelques pieds de Chêne pédonculé (Q. pedunculata) dans le fond du vallon où l'eau stagne quelquefois et où se montrent de larges taches de Carex brizoides. Ce Chêne rouvre est de très mauvaise qualité : gélif ou étoilé. Il occupe presque seul le versant sableux.

Le Hêtre (Fagus silvatica) est rare dans l'ensemble: il est inexistant dans les parties sableuses ou humides; on ne le rencontre en quantité appréciable que sur le versant argileux exposé au N.O. Cette répartition paradoxale (en effet le Hêtre préfère généralement les sols bien drainés à ceux qui sont compacts) s'explique aisément par une humidité trop faible dans le sable.

Le Charme (Carpinus betulus) et le Tilleul à petites feuilles ( $T$ lia sylvestris) ne sont abondants que par place.

Le Bouleau (Betula alba) est très répandu comme il convient dans un peuplement clair; il est remplacé par le Tremble (Populus tremula) dans le bas-fond.

Tous les fruitiers (Rosacées) sont très rares, ce qui est normal en sol acide.

Le Robinier (Robinia pseudoacacia) a été planté dans le fond du vallon, station qui se trouve être très semblable à celles où on le rencontre dans son pays d'origine. Il pousse très vigoureusement, mais il pourrit de bonne heure.

Le Pin sylvestre (Pinus silvestris) a été introduit en petite quantité: peu de sujets sont beaux, bien que la station doive en principe convenir à cette essence. Elle se couvre de lichens, a un rhytidome très épais et ne semble pas devoir être longévive.

La flore arbustive est encore plus pauvre; elle comprend le Coudrier (Corylus avellana), la Bourdaine (Rhamnus frangula) fréquents, le Genêt (Sarothammus scoparius) et la Bruyère (Calluna vulgaris) rares.

Densité et hauteur. - La densité est faible en raison de la mauvaise qualité de la station, laquelle élimine un grand nombre d'essences de remplissage, spécialement les essences d'ombre, les calcicoles et les fruitiers. Dans les plus mauvaises parties on trouve 
une futaie claire de petits Chênes à aspect souffreteux, à fût court et déprécié par des gourmands, recouvrant un taillis qui à l'âge de 30 ans est à la fois clair, court, grêle et à demi-mort. C'est presque normal pour les cépées de Chêne avide de lumière, c'est extraordinaire pour le Coudrier qui se plait d'habitude parfaitement sous le couvert du Chêne. Quant aux pieds de Hêtre rencontrés çà et là, ils donnent l'impression de se maintenir avec peine et non de s'accroître.

Dans les argiles un peu draînées, le Hêtre assez vigoureux tend à éliminer les essences de lumière et sa forme défectueuse peut être imputée au mode de traitement subi par la forêt: il est de réintroduction naturelle récente, par pieds isolés qui se sont installés sous des Chênes par lesquels ils furent longtemps dominés.

\section{CONCLUSIONS PROVISOIRES DU PRATICIEN}

Réduit à ses seuls moyens d'observation, le forestier ne peut expliquer pourquoi une pareille forêt est improductive: le climat ne présente aucune particularité remarquable.

Le sol est profond et bien rares sont les terrains dont la pauvreté ne permet pas à une forêt de pousser convenablement.

La dégradation du bois du Prieur est plus grande que celle des massifs contigus dont les vicissitudes anciennes ont dî̀ être à peu près les mêmes: ceci donne à penser qu'il s'agit d'un phénomène hl:main relativement récent et sans doute partiellement réversible. La portion argileuse de ce bois semble soumise à une évolution progressive, tandis que le surplus voit tout juste sa régression -stabilisée.

La très grande abondance, malgré sa mauvaise qualité, d'un Chêne rouvre aujourd'hui sans valeur s'explique par l'histoire générale des forêts comtoises (parcours des porcs et des bêtes à cornes, gros besoins en combustible et fortes coupes de bois à courte révolution) ainsi que par la médiocrité de la station (faible concurrence vitale). L'extraordinaire fréquence de la gélivure indiquerait une carence très prononcée en bases (calcium principalement).

Aucune essence forestière courante ne parait donner de résultats intéressants dans la zone sablonneuse, alors que le Hêtre, avec le temps, réussira sans doute à tirer parti du versant argileux.

\section{Etude pédologique}

Résolu à en avoir le cœur net, le propriétaire se livra à une étude approfondie du sol: il ouvrit trois profils en trois points aussi typiques que possible, effectua des prélèvements que le Laboratoire de Pédologie de la Station de Recherches de Nancy vou- 
lut bien lui analyser. Nous en exprimons ici à son Directeur toute notre gratitude.

Les résultats fournis par les méthodes physico-chimiques ( $\mathrm{Ph}$. Duchaufour, I et 2) donnent l'acidité, la composition granulométrique et la richesse en principes fertilisants. Ces derniers représentent non pas la quantité totale contenue dans le sol, mais pour les bases les quantités échangeables, pour l'acide phosphorique la partie assimilable. Seul l'azote a été dosé en entier. Il y a donc là encore une cause d'incertitude: on sait en effet que les microorganismes du sol sont capables d'attaquer et de solubiliser des éléments minéraux réputés inassimilables pour les végétaux supérieurs. Nous y'reviendrons d'ailleurs plus loin.

\section{Profil N ${ }^{\circ}$ I (FI)}

Versant S.E. - pente de $20 \%$ - sol très sablonneux - Futaie claire de Chêne rouvre à peu près pur, recouvrant une souille de Coudriers et de quelques Hêtres.

Couverture vivante: à côté d'une flore ubiquiste assez pauvre, on rencontrei une flore de sol modérément acide: Pteris aquilina, Lonicera periclymenum, Convallaria maialis, Melampyrum sylvaticum, Hypnum triquetrum en grande quantité, mais aussi Anemone nemorosa.

On aperçoit successivement dans le sol:

- une couverture morte peu importante,

- un horizon $\mathrm{A}_{1}$ épais de quelque's centimètres, très noir,

- u11 horizon $A_{2}$, de quelques centimètres également, que l'on pourrait qualifier de micropodzol, de couleur grise avec de nombreux grains de quartz blanc.

- on trouve en dessous un sable ocreux de moins en moins foncé que l'on prendrait pour la roche-mère si l'on ne découvrait plus bas des taches couleur de rouille permettant de dire que les niveaux $\mathrm{B}$ et $\mathrm{C}$ sont confondus.

- les racines s'arrêtent vers $-0,3 \mathrm{~m}$; le sable devient plus compact vers $-0,6 \mathrm{~m}$ et vers - I m apparaissent des taches couleur de rouille.

$\mathrm{La}$ fouille s'est arrêtée vers - $\mathrm{I}, 2 \mathrm{~m}$.

Le tableau suivant résume les résultats de l'analyse faite par la Station de. Recherches:

Analyse physique

\begin{tabular}{|c|c|c|c|c|c|c|c|}
\hline Profondeur & $\mathrm{pH}$ & $\begin{array}{c}\text { Argile } \\
\%\end{array}$ & $\begin{array}{c}\text { Limons } \\
\%\end{array}$ & $\begin{array}{c}\text { Sables } \\
\text { fins } \\
\%\end{array}$ & $\begin{array}{c}\text { Sables } \\
\text { grossiers } \\
\%\end{array}$ & $\begin{array}{c}\text { Mat. } \\
\text { org. } \\
\%\end{array}$ & $\begin{array}{r}\text { Eau } \\
\%\end{array}$ \\
\hline - & - & - & - & - & - & - & - \\
\hline$-0, \mathrm{I} \mathrm{m}$ & 4,8 & 5 & 8 & 38 & $46, \mathrm{I}$ & $2, \mathrm{I}$ & 0,8 \\
\hline$-0,3 \mathrm{~m}$ & 5,3 & 2,5 & 9 & 36,8 & 50,9 & 0,3 & 0,5 \\
\hline$-\mathrm{I}, \mathrm{O} \mathrm{m}$ & 5,5 & 7 & II & $4 \mathrm{I}, 9$ & 39,2 & 0,2 & 0,7 \\
\hline
\end{tabular}


Analyse chimique

$\begin{array}{cccccccc}\text { Profondeur } & \mathrm{C} \% & \begin{array}{c}\text { Fer } \\ \text { libre \% }\end{array} & \mathrm{Ca} & \mathrm{K} & \mathrm{P}_{2} \mathrm{O}_{5} & \mathrm{~N} \% & \mathrm{C} / \mathrm{N} \\ - & - & - & - & - & - & - & - \\ -0,1 \mathrm{~m} & \mathrm{I}, 23 & 0,29 & 0,18 & 0,1 \mathrm{I} & 0,01 & 0,08 & 15,4 \\ -0,3 \mathrm{~m} & 0,18 & 0,50 & 0,23 & 0,07 & 0,01 & - & - \\ -\mathrm{r}, 0 \mathrm{~m} & 0,14 & 0,65 & 0,68 & 0,09 & 0,02 & - & -\end{array}$

Note: Ca et $\mathrm{K}$ sont exprimés en milliéquivalents pour Ioo grammes (I équivalent $\mathrm{Ca}=20 \mathrm{gr}$; I équiv. $\mathrm{K}=39 \mathrm{gr}$.)

I1 saute aux yeux que l'on a affaire à un sol extrêmement perméable dans lequel les éléments fins ont été entraînés en profondeur; naturellement très pauvre, il s'est vu encore appauvri en surface par un lessivage facile. Néanmoins, l'acidité n'est pas excessive, les matières organiques ne s'accumulent pas et le rapport $\mathrm{C} / \mathrm{N}$ est assez favorable.

Le laboratoire de Nancy a diagnostiqué un sol à Moder.

\section{Profil $\mathrm{N}^{0} 2(\mathrm{~F} 2)$}

Situé presque au fond du vallon; pente: $5 \%$; même sol qu'au profil $n^{\circ}$ I.

Futaie claire de Chênei moitié rouvre, moitié pédonculé. Taillis comprenant surtout du Charme mélangé de Coudrier, de Tremble. Couverture vivante: comme plus haut, avec en plus Hedera helix. Couverture morte peu épaisse.

L'horizon $A_{1}$ est comme plus haut, mais avec moins de grainst blancs.

La.fouille, conduite jusqu'à - I,I m n'a pas décelé de taches ferrugineuses; donc, ou bien le fer est descendu plus bas, ou bien le sable ocreux constitue indiscutablement la roche-mère. La pioche n'a pas senti d'augmentation de la compacité vers $-0.6 \mathrm{~m}$ comme au profil I.

Analyse physique

\begin{tabular}{|c|c|c|c|c|c|c|c|}
\hline Profondcur & $\mathrm{pH}$ & $\begin{array}{c}\text { Argile } \\
\%\end{array}$ & $\begin{array}{c}\text { Limons } \\
\%\end{array}$ & $\begin{array}{c}\text { Sables } \\
\text { fins } \\
\%\end{array}$ & $\begin{array}{c}\text { Sables } \\
\text { grossiers } \\
\%\end{array}$ & $\begin{array}{c}\text { Mat. } \\
\text { org. } \\
\%\end{array}$ & $\begin{array}{c}\text { Eau } \\
\%\end{array}$ \\
\hline - & - & - & - & - & - & - & - \\
\hline$-0, \mathrm{I} \mathrm{m}$ & 5,4 & 4 & 7 & 31,6 & 54,4 & 2,3 & 0,7 \\
\hline$-0,3 \mathrm{~m}$ & 5,6 & 2 & 7 & 34,5 & 55,6 & 0,3 & 0,6 \\
\hline$-\mathrm{I}, \mathrm{O} \mathrm{m}$ & 5,4 & I, 5 & 7,5 & 33 & 57,3 & $0, \mathrm{I}$ & 0,6 \\
\hline
\end{tabular}

Analyse chimique

$\begin{array}{cccccccc}\text { Profondeur } & \mathrm{C} \% & \begin{array}{c}\mathrm{Fer} \\ \text { libre } \%\end{array} & \mathrm{Ca} & \mathrm{K} & \mathrm{P}_{2} \mathrm{O}_{5} & \mathrm{~N} \% & \mathrm{C} / \mathrm{N} \\ - & - & - & - & - & - & - & - \\ -0,1 \mathrm{~m} & \mathrm{I}, 32 & 0,32 & 0,72 & 0,15 & 0,01 & 0,08 & 16,5 \\ -0,3 \mathrm{~m} & 0,17 & 0,40 & 0,23 & 0,09 & 0,02 & - & - \\ -\mathrm{r}, 0 \mathrm{~m} & 0,06 & 0,45 & 0,30 & 0,07 & 0,01 & - & -\end{array}$


Le $2^{\mathrm{\theta}}$ sol est encore plus pauvre en éléments fins que le $\mathrm{I}^{\mathrm{er}}$; malgré cela l'entrainement du fer en profondeur est moindre. Quant au Ca, non seulement il n'a pas été lessivé, mais il est remonté en surface avec les feuilles tombées des arbres.

La Station de Recherches qualifie ce sol de sol brun à 'Mull. La présence du charme et du chêne pédonculé est expliquée par la richesse relative en calcium, ce qui confirme toutes les observations faites par les praticiens. Là se trouve la raison de la plus grande facilité des conversions en futaie sur sol acide: il n'y a pas à lutter contre le Charme qui empoisonne les forêts en sol calcaire.

\section{Profil $\mathrm{N}^{\circ} 3(\mathrm{~F} 3)$}

En haut du versant exposé au N.O. - pente très faible - sol d'argile ocre rouge mêlée de quelques graviers siliceux.

Futaie claire composée de 7/ı Chêne rouvre et 3/ıo Hêtre avec des taches de Bouleaux, de Tremble. Souille de Hêtre et dee Bourdaine.

Couverture vivante: Pteris aquilina. Lonicera periclymenum, Rubus, Polytrichum. La fraîcheur du sol est marquée par la présence de Mnium et d'Hépatiques.

Couverture morte: peu abondante.

Vers $-0,3 \mathrm{~m}$ apparaissent des taches de rouille (marmorisation), et vers $-0,5 \mathrm{~m}$ des taches livides ou gris bleu. Let pseudogley est très net à $-0,7 \mathrm{~m}$.

Analysei physique

\begin{tabular}{|c|c|c|c|c|c|c|c|}
\hline Profondeur & $\mathrm{pH}$ & $\begin{array}{c}\text { Argile } \\
\%\end{array}$ & $\begin{array}{c}\text { Limons } \\
\%\end{array}$ & $\begin{array}{c}\text { Sables } \\
\text { fins } \\
\%\end{array}$ & $\begin{array}{c}\text { Sables } \\
\text { grossiers } \\
\%\end{array}$ & $\begin{array}{l}\text { Mat. } \\
\text { org. } \\
\%\end{array}$ & $\begin{array}{c}\text { Eau } \\
\%\end{array}$ \\
\hline - & - & 一 & - & - & - & - & - \\
\hline$-0, \mathrm{I} \mathrm{m}$ & 5 & I2 & 33,5 & $38, \mathrm{I}$ & $\mathrm{I} 3,4$ & $\mathrm{I}, 6$ & $\mathrm{I}, 4$ \\
\hline$-0,3 \mathrm{~m}$ & $5, \mathrm{I}$ & 23,5 & 30 & 34,2 & 9,3 & 0,4 & 2,6 \\
\hline$-0,7 \mathrm{~m}$ & 4,9 & $4 \mathrm{I}$ & I9 & 26 & 9,5 & 0,4 & $4, \mathrm{I}$ \\
\hline
\end{tabular}

Analyse chimique

$\begin{array}{cccccccc}\text { Profondeur } & \mathrm{C} \% & \begin{array}{c}\mathrm{Fer} \\ \text { libre } \%\end{array} & \mathrm{Ca} & \mathrm{K} & \mathrm{P}_{2} \mathrm{O}_{5} & \mathrm{~N} \% & \mathrm{C} / \mathrm{N} \\ - & - & - & - & - & - & - & - \\ -0, \mathrm{I} \mathrm{m} & 0,95 & 0,46 & 0,26 & 0,19 & 0,0 \mathrm{I} & 0,06 & 15,8 \\ -0,3 \mathrm{~m} & 0,26 & 2,0 & 0,18 & 0,14 & \text { traces } & - & - \\ -0,7 \mathrm{~m} & 0,23 & 2,04 & \mathrm{I}, \mathrm{I0} & 0,24 & 0,0 \mathrm{I} & - & -\end{array}$

Sol fortement lessivé, à sous-sol très imperméable.

Malgré la présence d'argile et de Mousses indiquant une station assez frâ̂che, c'est le Chênel rouvre que l'on rencontre en raison de la pauvreté en calcium des couches accessibles aux racines.

\section{Enseignements de L'Analyse Physico-Chimioue}

En aucun cas l'acidité n'est excessive: pas de trace de myrtille, quelques coussinets de Leucobryum glaucum et de Dicranum dans la station $\mathrm{n}^{\circ} 3$. 
Le sol est plutôt profond dans les stations I et 2 ; il l'est encore suffisamment dans la station $\mathrm{n}^{\circ} 3$ à pseudo-gley.

L'insolation du versant S.E. combinée à la très grande perméabilité du terrain peut amener une disette en eau certaines années.

Mais c'est la composition chimique, du moins celle donnée par les méthodes d'analyse utilisées, qui semble offrir la meilleure explication du mauvais état de végétation des peuplements: en effet, la forêt de Fédry (station $\mathrm{n}^{\mathrm{o}}$ I) marque une proche parenté avec celle de Bruyères dont le sol brun podzolique a été décrit par $\mathrm{Ph}$. Duchaufour (2); la formation végétale correspondante est une rapaille à Pin sylvestre et taillis dégradé de Chêne rouvre.

La solution qui s'impose au propriétaire à la lumière de ces recherches, paraît être la substitution d'essences frugales à des essences feuillues exigeantes, en ayant soin d'éviter l'emploi des Pins qui dégraderaient davantage encore le sol.

\section{Etude microbiologique}

Toutefois, avant de reconvertir sa forêt, le propriétaire a voulu avoir l'avis du microbiologiste. En effet, les microorganismes du sol peuvent rendre de signalés services au forestier:

$\mathrm{I}^{\mathrm{o}}$ en dégradant la couverture morte, ils l'empêchent de s'accumuler et de devenir gênante: ils semblent remplir parfaitement cet office à Fédry et l'on devra s'attenclre à trouver dans le sol un grand nombre d'organismes cellulolytiques.

$2^{\circ}$ la richesse du sol en azote total est plutôt faible: c'est que les microorganismes fixateurs remplissent mal leur office; peut-être pourrait-on remédier à cet état de chose, non pas en répandant de coûteux engrais qui disparaîtraient rapidement en profondeur à travers le sable, mais en activant les sources gratuites d'azote.

$3^{\circ}$ on peut penser que le sol est plus riche en bases qu'il n'apparaît à l'analyse physico-chimique: c'est surtout probable clans la station $n^{\circ} 3$ très argileuse. On sait que le fer, la potasse et la chaux, ainsi d'ailleurs que l'acide phosphorique, peuvent être libérés par certains microorganismes à partir de composés insolubles.

Serait-il possible, en apportant au besoin de petites quantités d'engrais agissant comme catalyseurs, d'activer la partie utile de la microflore?

\section{LA MICROPOPULATION DES SOLS}

Et tout d'abord de quoi se compose cette microflore qui, avec la macroflore - les végétaux supérieurs - et la microfaune, fait du sol un véritable organisme vivant très complexe?

Les BActéries en représentent habituellement le groupe le plus nombreux: il s'agit de Bactéries aérobies ou anaérobies, c'est-à- 
dire vivant de préférence en absence d'oxygène, formant ou non des spores - autrement dit des organes de résistance.

Les exigences nutritives des unes et des autres sont très variables (voir les travaux du Canadien Locuniead), de même que leurs aptitudes physiologiques: certaines espèces participent au métabolisme azoté du sol (germes ammonifiants, fixateurs d'azote atmosphérique: Azotobacter aérobies ou Clostridium plus ou moins anaérobies, etc...), d'autres à la destruction de la matière organique clu sol (protéolytiques, pectino- et cellulolytiques par exemple), etc...

Ces mêmes Bactéries possèdent souvent plusieurs cordes à leur arc: les Azotobacter peuvent ainsi fixer l'azote de l'air, mais aussi fabriquer des substances de croissance clu type de l'acide indolacétique (auxine).

Le nombre des formes anaérobies est relativement faible: d'après E.N. Misiroustine (3) résumant de nombreux travaux concernant les sols d'U.R.S.S., il ne dépasserait pas d'hahitude ro \% de la population microbienne des sols.

Viennent ensuite les Actinomycètes qui peuvent être considérés comme des formes intermédiaires entre les Bactéries et les Champignons inférieurs. Le genre le plus connu en est le g. Streptomyces. Les Actinomycètes possèdent tous des propriétés chimiques très variables avec les espèces, mais toujours importantes, particulièrement en ce qui concerne la formation de l'bumus et leurs effets antagonistes envers les autres micro-organismes du sol. En effet, ils synthétisent des antibiotiques puissants et des matières colorantes qui donnent en partie leur couleur aux composés humiques.

Tous les grands groupes de Cirampignons sont représentés dans les sols: Phycomycètes (qui groupent entre autres les Mucor, Mortierella, etc...), les Ascomycètes (Penicillium, Aspergillus, etc...), Basidiomycètes supérieurs et Fungi Imperfecti (Trichoderma, Fusarium). Ils produisent un nycélium important, des spores nombreuses et prédominent souvent dans les terrains incultes ou à $\mathrm{pH}$ bas. La répartition des espèces paraît être liée au climat (MishousTINE).

Les Algues (Cyanophycées, Diatomées, Chlorophycées) vivent à la surface du sol, indépendamment de la matière organique, du fait de leur pouvoir assimilateur

Un autre groupe important enfin est constitué par les ProtozOAIRES, surtout abondants dans les sols organiques humides. Bien entendu, les Virus et les Bactériophages ne sont pas absents et peuvent jouer un rôle de premier plan (vis-à-vis des Bactéries symbiotiques des Légumineuses par exemple).

Par ces quelques traits descriptifs, nous voyons donc l'importance exceptionnelle de la micropopulation pour la vie des sols et leur fertilité. 
Cette fertilité est en rapport direct avec l'activité microbiologique qui s'y manifeste; on a le droit d'interpréter ce fait, soit comme une relation de cause à effet, soit comme une concomittance. On peut également dire que la microflore est d'autant plus abondante que le sol est plus riche, mais que l'action de la microflore se traduit par une plus grande fertilité du sol: on affirme ainsi l'interaction des facteurs de production. Lorsqu'on se propose d'agir sur un sol, il est indispensable de connaitre non seulement le rôle de chaque groupe fonctionnel, mais aussi les besoins des différents organismes de façon à pouvoir diriger l'évolution dans un sens favorable à l'homme.

Imaginons un propriétaire forestier à la hauteur de sa tâche: au vu d'une analyse physico-chimique, puis biologique de ses sols:

$I^{\circ}$ il décidera d'essayer de favoriser les éléments fortement antibiotiques, de façon à purger le sol de sa forêt d'éléments indésirables. Est-ce vraiment possible? Nos connaissances sont encore peu avancées sur ce point. Il y a lieu toutefois de rappeler la pratique des forestiers U.S.A. qui favorisent le Trichoderma viride pour éviter la pourriture des bois stockés.

$2^{\circ}$ il cherchera à développer les agents fixateurs d'azote en leur faisant peut-être un prêt de démarrage sous forme d'un petite quantité de $\mathrm{Ca}$ ou de $\mathrm{P}$, dépense qui lui sera rendue au centuple plus tard.

$3^{\circ}$ la comparaison entre l'analyse chimique globale de $\mathrm{Ca}, \mathrm{K}$ et $\mathrm{P}$, et le dosage par les méthodes classiques de la partie réputée assimilable des mêmes éléments a mis parfois en évidence une réserve inutilisable dans les conditions actuelles, mais mobilisable par certains microorganismes : nous précisons immédiatement qu'il ne s'agit pas là d'une hypothèse aventurée, mais d'une réalité démontrée. Il faut avoir vu des Lichens corroder en quelques dizaines d'années une plage de granite parfaitement polie pour acquérir la conviction que nos connaissances ont bien besoin d'être complétées. Cependant des travaux récents nous montrent l'exceptionnelle importance de ces germes. LASZLO, par exemple, a pu isoler certains microbes et prouver leur aptitude à libérer le $\mathrm{P}_{2} \mathrm{O}_{5}$ de phosphates insolubles tels que l'apatite. Cultivant ensuite du Blé et des petits Pois dans des pots de culture ne contenant pas de phosphore autre que celui contenu dans l'apatite, il garde certains pots comme témoins, en ensemence d'autres avec les germes qu'il a pu isoler. Après un certain temps de croissance, les plants prélevés dans la deuxième série de pots voient leur poids doublé par rapport au témoin (4).

Il sera tentant de favoriser spécialement ces auxiliaires pour offrir ces réserves aux végétaux supérieurs qui en manquent, faute de moyens d'attaque. 


\section{LES RÉSUltats DE L'ANALYSE MICROBIOLOGIQUE}

Mais passons en revue les différentes catégories de microorganismes qui vivent dans les sols étudiés. Pour le moment, nous ne pourrons sans doute que constater des différences entre les microflores de ces sols et y voir des sujets d'études ultérieures*.

Les résultats de nos analyses concernant la flore totale des trois sols de Fédry et les Champignons qui s'y développent sont exposés dans les schémas suivants. Nous aurons l'occasion de revenir ultérieurement sur ces sols et leur microflore, particulièrement sur leur flore fongique qui a été étudiée en collaboration avec W. Gams (Innbrück).

Les nombres de germes sont exprimés pour I gramme de terre séchée à $105^{\circ} \mathrm{C}$.

Flore du sol - Fédry.

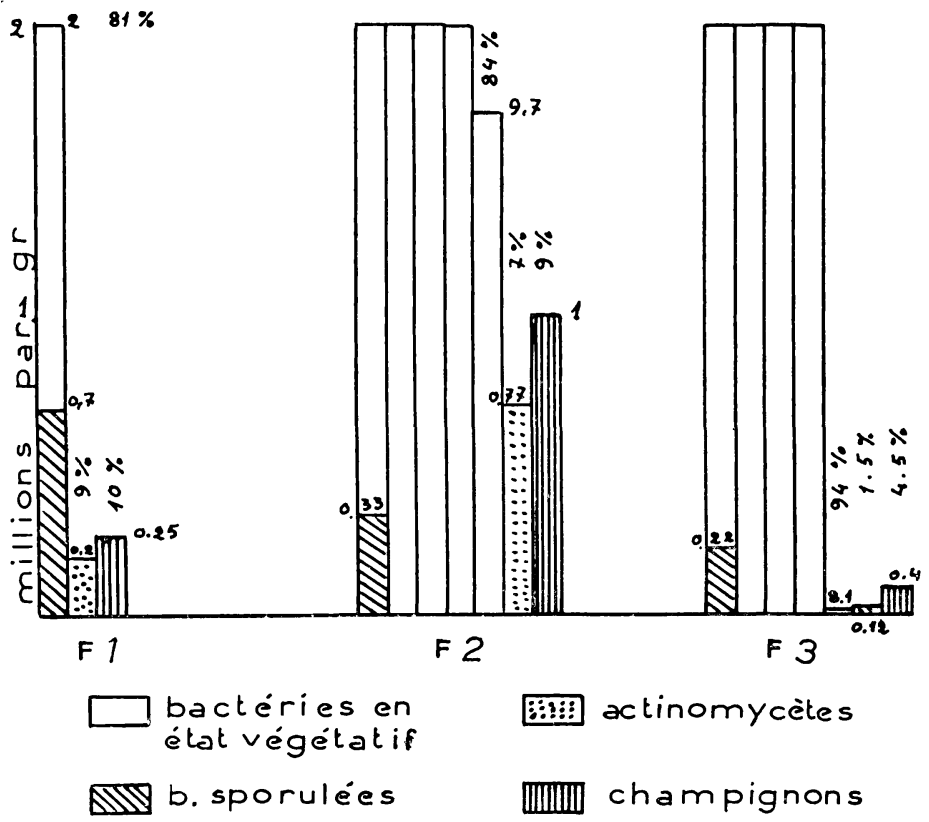

* Les méthodes employées sont celles en usage dans le laboratoire de l'un de nous. Elles seront d'ailleurs exposées en détail dans une publication prochaine. 


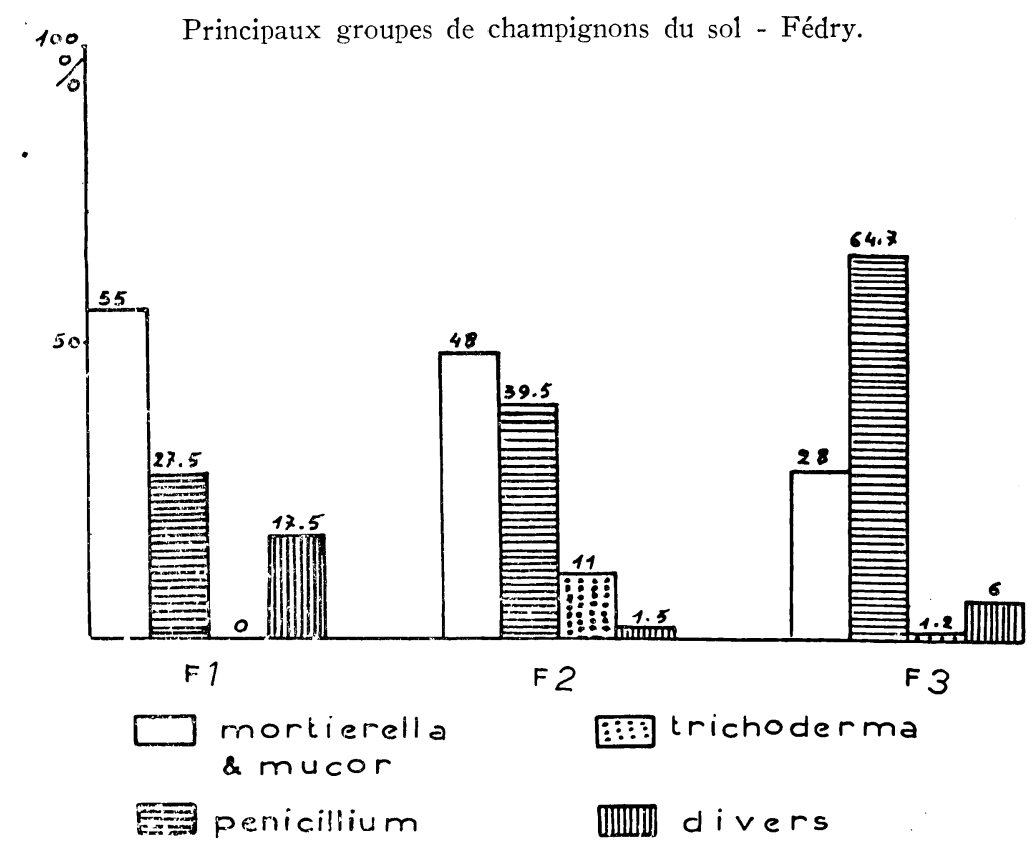

Nous voyons d'après ces figures que la microflore totale est très pauvre dans chacune de ces terres, la station F I étant la plus défavorisée et cclle dont la pauvreté est la moins cruelle, étant certainement F2. (Rappelons qu'un sol agricole peut renfermer au moins ıoo fois plus cle germes.)

Il est intéressant de noter que les pourcentages des trois groupes étudiés: Bactéries, Actinomycètes et Champignons, sont très semblables dans $F_{1}$ et $F_{2}$. Ces deux sols semblent très voisins du point de vue bactériologique, alors que $\mathrm{F}_{3}$ est nettement différent. Notons enfin, en comparant $\mathrm{F}_{2}$ et $\mathrm{F}_{3}$ que les Actinomycètes jouent un rôle plus important dans la première, alors que les Bactéries l'emportent dans la seconde station.

Les germes sporulés enfin sont très abondants en Fi. Dans les autres terres, ils sont au même point, le pourcentage étant le plus faible dans F3. Qu'en penser? Nous avons vu que les spores sont les " organes " de résistance de ces germes. On peut donc croire que schématiquement les Bactéries sporulées seront d'autant plus nombreuses que les conditions seront plus dures, comme c'est le cas ici.

Cette interprétation est admissible. Mais il est bon de se souvenir surtout que les Bactéries sporifères, comme les Actinomycètes d'ailleurs, pcssèdent un puissant appareil de fermentation et auront donc. une grande importance dans la décomposition de la matière orga- 
nique. Très sommairement, la destruction des composés humiques est le fait d'abord des Bactéries non sporifères et des Champignons. Les phases suivantes sont dominées par les Bacilles sporulés et les processus de dégradation sont achevés par les Actinomycètes qui détruisent le noyau cyclique des acides humiques (E.N. MishousTINE). Cela nous éclaire singulièrement : les Bactéries sporulées de Fi seront avant tout des agent de dégradation de la matière organique. Or, nous savons déjà que la couverture morte est très peu importante, dans les trois sols d'ailleurs; F I semble donc renfermer une " flore de catabolisme ) et non d'édification et de mobilisation. Il est plus difficile de se prononcer sur les deux autres stations dont la flore paraît mieux équilibrée.

Quant aux Champignons inférieurs, nous voyons que là encore $F_{1}$ et $F_{2}$ se ressemblent par la préínininance des Mucorales (Mortierella surtout et Mucor). Toutefois, on voit un virage s'amorcer avec $\mathrm{F}_{2}$ : les Penicillium ou voisins et Trichoderma sont plus abondants. $F_{3}$ nous montre un tableau qui est à l'inverse de $F_{I}$ : peu de Mucorales, Penicillium et apparentés nombreux.

Ajoutons que l'examen des germes isolés au cours de l'étude de la flore totale confirme les observations précédentes: la flore de FI est beaucoup plus une flore de consommation que de capitalisation; la majorité des germes exigent de l'azote organique pour leur cléveloppement: c'est le signe de sols où les processus de mobilisation sont faibles. F2 est l'inverse: les germes capables d'utiliser l'azote minéral y sont plus abondants. C'est, d'après Misiroustine toujours, le fait de sols à processus de mobilisation plus énergiques. Quant à $F_{3}$, son image est différente à la fois des deux précédentes.

Les germes antibiotiques enfin sont peu actifs dans tous les cas, particulièrement en $\mathrm{F}_{\mathrm{I}}$. A ce propos, il est bon de signaler qu'en août 1958, une tornade a parcouru la vallée de la Saône et renversé un grand nombre d'arbres; la région de Fédry n'a pas été épargnée et l'on a pu constater que dans la zone avoisinant le profil $\mathrm{n}^{\circ} \mathrm{I}$, à germes antibiotiques rares et peu actifs, les arbres renversés présentent de nombreuses traces de carie des racines. On peut penser que les Champignons de la pourriture se sont donné libre cours dans un secteur où rien ne les contrariait.

En ce qui concerne les Bactéries du cycle de l'azote, on ne peut relever que leur faible activité. Les Azotobacter sont absents, de même que les nitrificateurs. Les Clostridium sont peut actifs, sauf en F2. L'ammonification se fait bien, surtout en $F_{1}$ et $F_{2}$. Les processus d'ammonification se placent à la fin de la dégradation des albuminoïdes, ou protéines; mais il y a également formation d'ammoniaque au cours de la dénitrification qui est assez active dans ces trois sols. De toute façon, s'il n'existe pas d'organismes en suffisance pour récupérer l'ammoniaque dégagée, il en résultera pour le sol une perte en azote importante. 
Les agents bactériens de la cellulolyse sont pratiquement absents de $F_{3}$, d'une activité faible en $F_{1}$, mais assez forte en $F_{2}$. Par contre, la cellulolyse fongique est forte en $F_{I}$ et très forte en $F_{3}$, mais beaucoup plus faible en $\mathrm{F}_{2}$ ò̀ le Trichoderma viride représente l'espèce princinale.

Au total la cellulolyse est satisfaisante dans tous les cas, les Bactéries et les Champignons ayant des exigences opposées en matière d'acidité du milieu.

Dans les trois terres étudiées, nous n'avons pas observé de germes oxydant le soufre, ni de germes réduisant les sulfates. Par contre, il est nécessaire de mentionner les Bactéries du Fer. Elles sont connues depuis fort longtemps; cependant leur rôle dans les déplacements de ce métal est difficile à mettre en évidence. L'analyse chimique a montré une forte migration du fer dans les sols de Fédry, mouvement explicable certes par la porosité du terrain, mais aussi peut-être par une solubilisation bactérienne active: l'indice d'entraînement du fer est de 2 pour le premier profil et de 4 pour le dernier. Faute de temps, aucune culture spéciale de ces organismes n'a été réalisée; mais l'observation directe des flaques stagnant après de fortes pluies dans le vallon qui occupe le centre de la forêt, fait voir une épaisse couche irisée constituée par ces Bactéries. Après évaporation de l'eau, il reste sur le sol un dépôt couleur de rouille, assez important.

Il faut envisager enfin les agents susceptibles d'attaquer les phosphates insolubles. Ils se sont révélés actifs et abondants. Précisons que nous en avons fait l'étude, soit globalement sur un milieu au phosphate tricalcique (nilieu de PıкOwska modifié), soit souche à souche sur deux milieux différents: l'un étant également au phosphate tricalcique, l'autre contenant une source de phosphore organique insoluble (glycérophosphate). Les techniques sont celles de Mme J. Golfiniowska (5).

Sur le milieu de Prkowska, nous avons vu apparaitre surtout des Champignons en $\mathrm{F}_{\mathrm{I}}$ : Trichoderma viride — alors que dans l'étude spéciale des Champignons, cette espèce ne s'était pas manifestée -. des Morticrclla ( $M$. vinacea, p. ex.), Trichosporium, etc... Ensuite se sont développées d'importantes colonies d'Azotobacter.

En F2, le tahleau a été différent: nous avons noté de nombreuses colonies bactériennes appartenant à deux types différents, ainsi que des colonies fongiques, moins abondantes cependlant qu'en F $\mathrm{T}$ (Trichoderma viride surtout). A la suite de quoi les Azotobacter sont apparus, en association avec d'autres Bactéries.

Nous avons constaté des faits intermédiaires pour $\mathrm{F}_{3}$ : grandes quantités de Champignons (surtout des Mucorales, peu de Trichoderma) et de Bactéries. Tes Asotobacter se sont développés aussi, mais peu fortement. Ils étaient également associés à d'autres Bactéries. 
L'étude des germes isolés a révélé par contre un pourcentage beaucoup plus élevé de germes actifs en $\mathrm{F}_{2}$ et $\mathrm{F}_{3}$ que dans le premier sol.

\section{Conclusion de L'ANALYSE BACTÉRIOlOGIQUe}

A la suite de cette analyse détaillée, plusieurs points sont à relever :

- grande pauvreté et faible activité biologique de ces sols; ainsi que nous l'avons noté pius haut, leurs microflores - et particulièrement celle de $F_{I}$ - semblent être des flores de catabolisme, et non d'assimilation et de mobilisation: l'activité quasi nulle des différents groupes physiologiques - exception faite des cellulolytiques et des dénitrificateurs - en témoigne. Cependant, l'aptitude de nombreux germes surtout dans $\mathrm{F}_{2}$ et $\mathrm{F}_{3}$ à utiliser le phosphore insoluble, qu'il soit minéral ou contenu dans des composés organiques, constitue une potentialité intéressante.

- Une carence importante en phosphore soluble ou non et en calcium, déjà révélée par l'analyse chimique, est ici confirmée;

- un apport modéré au sol de phosphates même insolubles pourrait peut-être relancer des phénomènes aussi essentiels que la fixation de l'azote;

- les stations $F_{1}$ et $F_{2}$ sont assez semblables du point de vue microbiologique, $\mathrm{F}_{2}$ étant la mieux équilibrée et présentant l'activité biologique la plus importante. $\mathrm{F}_{3}$ montre une image fort différente des autres stations. Il apparait donc que chaque type de sol renferme une microflore particulière. Des rapports étroits semblent exister entre les sols, leur microflore et la végétation correspondante.

On voit par ces quelques renseignements, toute l'importance d'une fiche signalétique très détaillée du point de vue microbiologique, pour les recherches agricoles et forestières.

\section{Conclusion générale}

La forêt de Fédry repose sur un sol pauvre ou très pauvre, dégradé par des abus séculaires: la microflore est à la fois peu abondante et peu active. Les sources minérales d'éléments fertilisants sont maigres et la production d'azote assimilable est fort réduite. L'exploitation d'un peuplement forestier misérable s'avère déficitaire, compte tenu de la mévente des petits bois feuillus.

Il apparaît qu'une double série de mesures s'impose:

$I^{\circ}$ Enrésinement: de façon à cultiver des essences moins exigeantes que les feuillus et d'un meilleur rapport, même pour de petites dimensions. On aura soin de faire appel à des essences peu acidifiantes et par conséquent d'exclure les Pins et les Epicéas. Sui- 
vant la densité du peuplement existant et l'exposition, on introduira Psendotsuga, Cedrus, Abies numidica, Abies grandis, Abies nordmanniana, Abies alba.

$2^{\circ}$ Amélioration du sol: a) La solution la plus simple et la moins coûteuse, celle qu'imposent souvent les circonstances (revenu négatif, absence de main-d'œuvre), c'est le repos, la jachère. Ce procédé, vieux comme le monde, tombé aujourd'hui dans l'oubli, a permis à l'espèce humaine de survivre pendant des siècles. Alors que la terre se repose, les microorganismes reconstituent les réserves d'azote et solubilisent une nouvelle tranche de $\mathrm{Ca}, \mathrm{K}$ et $\mathrm{P}$ à partir des réserves minérales du sol.

b) Amélioration biologique: depuis longtemps on a recommandé en Europe Centrale le semis de Lupin; des pédologues belges ont attribué récemment au Genêt la propriété de transformer les podzols. Le gros inconvénient de ces méthodes, c'est, outre leur prix de revient, la faible durée des plantes introduites dans un massif fermé. La prolifération du Genêt après les coupes abusives pourrait être une réaction de défense de la forêt.

c) Apport d'engrais chimiques: La question est à l'ordre du jour un peu partout: c'est une solution énergique mais côteuse, et sa rentabilité n'est pas assurée dans un pays où la propriété forestière ne jouit pas de la bienveillance des pouvoirs publics.

On ignore encore presque tout de la réaction d'un sol forestier à un apport massif d'engrais ou d'amendement; on commence à se douter des dangers présentés par le chaulage qui risque de détruire ou de paralyser de nombreux microorganismes, et parmi les plus actifs, générateurs d'antibiotiques puissants et favorables. On a aussi la certitude de voir disparaitre en profondeur tous les sels solubles. On peut penser que la forêt de Fédry profiterait d'une addition de scories potassiques à la terre des potets: on l'enrichirait ainsi en chaux, potasse et phosphore dont les plants introduits bénéficieraient immédiatement. En profiteraient également les Légumineuses fixatrices d'azote; on activerait sans doute aussi la microflore, mais dans un sens ignoré que l'on peut espérer favorable. L'assimilation de l'azote alors reprendrait une certaine vigueur dans ces sols moribonds et par conséquent leur rendrait quelque fertilité.

Des essais sont faits à tâtons qui auraient besoin d'une base scientifique solide.

Cela nous permet d'insister une fois de plus sur la nécessité de ne pas isoler la pédologie du contexte biologique, ni la biologie du sol de son support chimique.

\section{R. SchaEfFER, \\ Eaux et Forêts, Bessançon.}

R. Moreau,

Ecole Nationale de Médecine et de Pharmacie, Besançon. 


\section{BIBLIOGRAPHIE}

I. Duchaufour (Ph.). - (I956) Pédologie. Applications forestières et agricoles. I vol., 310 p., Nancy.

2. Duchaufour (Ph.). - (I957) Pédologie. Tableaux descriptifs et analytiques des sols. I vol., 87 p., Nancy.

3. Mishoustine (E.-N.). - (I956) La science des associations microbiennes du sol ret son développement. VI Congrès de la Sci. du Sol. Paris, I956, III, 24, pp. I45-I50.

4. Laszlo (G.) et PoP (C1.). - (I957) La mobilisation des Phosphates par certaines espèces de micro-organismes. Pédologie, VII, $n^{\circ}$ spéc. (Symp. Méth. Et. Microbiol. Sol), pp. I72-180.

5. Golebrowska (J.). - (I956) A method of investigation of the microbial population playing a part in the transformation of phosphorus compounds in the soil. Acta Microbiologica Polonica, 5, pp. I25-I27. 